\title{
Fast Network Oscillations in the Hippocampal CA1 Region of the Behaving Rat
}

\author{
Jozsef Csicsvari, Hajime Hirase, András Czurkó, Akira Mamiya, and György Buzsáki \\ Center for Molecular and Behavioral Neuroscience, Rutgers, The State University of New Jersey, Newark, New Jersey \\ 07102
}

This study examined intermittent, high-frequency $(100-200 \mathrm{~Hz})$ oscillatory patterns in the CA1 region of the hippocampus in the absence of theta activity, i.e., during and in between sharp wave (SPW) bursts. Pyramidal and interneuronal activity was phase-locked not only to large amplitude ( $>7$ SD from baseline) oscillatory events, which are present mainly during SPWs, but to smaller amplitude ( $<4 \mathrm{SD}$ ) patterns, as well. Large-amplitude events were in the 140-200 Hz, "ripple" frequency range. Lower-amplitude events, however, contained slower, 100-130 $\mathrm{Hz}$ ("slow") oscillatory patterns. Fast ripple waves reversed just

Network patterns of the hippocampus include rhythms at theta $(5-10 \mathrm{~Hz})$ and gamma $(40-100 \mathrm{~Hz})$ frequencies observed in the rat during exploration and rapid eye movement sleep. In addition, intermittent population bursts are present in the CA3-CA1subiculum-entorhinal cortex axis during awake immobility, consummatory behaviors, and slow-wave sleep (Vanderwolf, 1969; Buzsaki et al., 1983; Bland, 1986; Stewart and Fox, 1990; Chrobak and Buzsaki, 1996). These intermittent population bursts are associated with sharp waves (SPW) in the CA1 stratum radiatum and reflect dendritic depolarization of CA1 pyramidal cells by the Schaffer collaterals and ultrafast $(140-200 \mathrm{~Hz})$ ripple oscillations in the pyramidal cell layer. The mechanisms of these fast oscillatory patterns in the hippocampus and neocortex are poorly understood (Buzsaki et al., 1992; Ylinen et al., 1995; Kandel and Buzsaki, 1997; Draguhn et al., 1998).

In between the large-amplitude fast ripple oscillations, the baseline activity is not flat, however, and the detection of ripple activity is somewhat arbitrary (Csicsvari et al., 1999). It is possible that smaller-amplitude and/or slower-frequency events and largeamplitude ripples reflect a continuum and are generated by the same mechanism. In this case, a weaker afferent drive would result in a qualitatively similar but slower frequency and loweramplitude fast oscillation. Alternatively, ripples may have a "threshold," and the field oscillations, present between largeamplitude ripple events, are brought about by a different mechanism. The present findings support the latter alternative.

\section{MATERIALS AND METHODS}

Animals, surgical procedures, and data acquisition methods were the same as described in previous papers (Ylinen et al., 1995; Csicsvari et al.,

Received Jan. 22, 1999; revised March 15, 1999; accepted March 24, 1999.

This work was supported by National Institutes of Health Grants NS34994 and MH54671 and the Human Frontier Science Program (to H.H.).We thank Darrell Henze for comments on this manuscript.

Correspondence should be addressed to György Buzsáki, Center for Molecular and Behavioral Neuroscience, Rutgers University, 197 University Avenue, Newark, NJ 07102.

Copyright (C) 1999 Society for Neuroscience $\quad 0270-6474 / 99 / 190001-\bullet \$ 05.00 / 0$ below the CA1 pyramidal layer, whereas slow oscillatory potentials reversed in the stratum radiatum and/or in the stratum oriens. Parallel CA1-CA3 recordings revealed correlated CA3 field and unit activity to the slow CA1 waves but not to fast ripple waves. These findings suggest that fast ripples emerge in the CA1 region, whereas slow $(100-130 \mathrm{~Hz})$ oscillatory patterns are generated in the CA3 region and transferred to the CA1 field.

Key words: sharp waves; ripples; gamma activity; inhibition; EEG; interneurons

1999). Briefly, 19 male rats were implanted with wire tetrodes or silicon electrode arrays to record unit and local field potential activity. Wire tetrodes were constructed from $(13 \mu \mathrm{m})$ polyimide-coated nichrome wires. Single-shank, 16 site silicon probes $(100 \mu \mathrm{m}$ recording site separation) were used to measure depth profiles of field activity $(n=3$ animals). Electrodes were attached to multidrives that enabled the independent movement of electrodes into the hippocampus. Commissural stimulating electrodes (anteroposterior, -0.8; lateral, 0.5; ventral, -4.2) helped determine the location of the electrodes. Unit activity and field potentials were recorded continuously using 16-channel ISC-16 analogto-digital boards (RC Electronics, Santa Barbara, CA) and a 64-channel DATAMAX system (RC Electronics). Data were stored on 12 gigabyte DDS-3 digital audio tapes and analyzed on $300 \mathrm{MHz}$ P-II system running under the Linux operating system. Recording and spike clustering procedures and the classification of pyramidal cells and interneurons also have been described before in detail (Csicsvari et al., 1999).

For the extraction of fast oscillatory events, the wide-band $(1 \mathrm{~Hz}-5$ $\mathrm{kHz})$ recorded data were digitally bandpass-filtered $(80-250 \mathrm{~Hz})$. The power (root mean square) of the filtered signal was calculated for each electrode and summed across electrodes to reduce variability. The power was calculated by summing squared sample values within a $1.6 \mathrm{msec}$ window and calculating the square root of this sum. The mean and SD of the power signal were calculated to determine the detection threshold. Oscillatory epochs with a power of $\geq 2$ SD above the mean were detected. The beginning and the end of oscillatory epochs were marked at points at which the power reduced to $<1 \mathrm{SD}$. Theta periods, detected by using the theta/delta power ratio (Csicsvari et al., 1999), were excluded from the analysis. Positive and negative peaks of individual waves were detected within the detected oscillatory epochs. Only waves with $>1$ SD peak amplitude, relative to the mean, were detected.

This article is published in The Journal of Neuroscience, Rapid Communications Section, which publishes brief, peerreviewed papers online, not in print. Rapid Communications are posted online approximately one month earlier than they would appear if printed. They are listed in the Table of Contents of the next open issue of JNeurosci. Cite this article as: JNeurosci, 1999, 19:RC20 (1-4). The publication date is the date of posting online at www.jneurosci.org.

http://www.jneurosci.org/cgi/content/full/3298 




Figure 1. Depth profile of hippocampal fast $(100-200 \mathrm{~Hz})$ oscillatory patterns during slow-wave sleep. A, Depth profile of a sharp wave (double arrow) and sharp wave-associated fast $(140-200 \mathrm{~Hz})$ ripple waves. $B$, Depth profile of a slower $(100-130 \mathrm{~Hz})$ oscillatory event. $A, B$, Single events recorded by a 16 -site silicon probe $(100 \mu \mathrm{m}$ recording site separation). Arrows mark the approximate site of phase reversal. Double arrow, Largest-amplitude SPW recorded in the stratum radiatum. Dashed lines mark the time of the largest negative oscillatory peak in the CA1 pyramidal layer. Displayed layers: $p$, CA1 stratum pyramidale; $o$, CA1 stratum oriens; $r$, CA1 stratum radiatum.

\section{RESULTS}

The amplitude of fast ripple waves was largest in the CA1 pyramidal layer. These large-amplitude ( $>7 \mathrm{SD}$ from background), fast $(>140 \mathrm{~Hz})$ ripples were typically associated with a negative sharp wave in the stratum radiatum (Fig. $1 A$ ). The fast ripple waves reversed abruptly below the pyramidal layer, and their amplitude decreased steeply in stratum radiatum. In between large ripples, the fast $(>100 \mathrm{~Hz})$ oscillatory field activity was relatively small or entirely absent (Fig. 1). These lower-amplitude events were of slower frequency $(<140 \mathrm{~Hz})$, and the oscillatory waves showed a phase reversal in either the stratum oriens (Fig. $1 B$ ) or stratum radiatum. Both types of oscillatory patterns displayed considerable variability in their length (i.e., the number of individual waves within an event) and amplitude distribution. These qualitative observations suggested that fast oscillatory waves in the CA1 region were generated by more than one mechanism.

The frequencies of the individual, high-frequency oscillatory epochs were best described by a bimodal distribution with peaks at 180 and $110 \mathrm{~Hz}$. The faster events were of larger amplitude than the "slow" (110 Hz) oscillations (Figs. 2, 3A). Both fast (180 $\mathrm{Hz}$ peak) and slow oscillations $(110 \mathrm{~Hz}$ peak) were associated with phase-locked discharge of both pyramidal cells and interneurons (Fig. 2). The amplitude of both fast and slow oscillatory events correlated with the relative increase in firing frequency of neurons (Fig. 3B,C). The correlation with pyramidal cell discharges was linear, except for the lowest-amplitude events $(<4$ $\mathrm{SD}$ ). Interneurons also increased their firing rate as a function of the field amplitude, but rate of the increase was considerably less than it was for pyramidal cells. Unit activity during the relatively flat $(<2$ SD) field epochs was virtually absent. Low-amplitude $(<4 \mathrm{SD})$ oscillatory events occurred on average five times more often than large-amplitude $(>4 \mathrm{SD})$ events $(<4 \mathrm{SD}, 3.73 \pm 0.193$ $\mathrm{Hz} ; n=53 ;>4 \mathrm{SD}, 0.73 \pm 0.036 \mathrm{~Hz} ; n=53)$.

The role of the CA3 region in the CA1 fast oscillatory events was investigated by simultaneous tetrode recordings from the CA1 and CA3 pyramidal layers. Wave-triggered averaging for the slow oscillatory event in CA1 revealed phase-locked field activity in the CA3 region (Fig. 4). In contrast, field activity in the CA3 pyramidal layer did not appear to be related to the individual waves of the fast ripples recorded in the CA1 field. In addition, cross-correlation between CA1 field activity and interneuronal discharge in the CA3 region revealed that unit discharges were phase-locked to the slow CA1 field activity but not with the large-amplitude, faster ripples $(n=4)$.

\section{DISCUSSION}

The findings of the present experiments indicate that fast oscillatory waves $(100-200 \mathrm{~Hz})$ in the hippocampal CA1 region can be induced by two different mechanisms. We suggest that fast ripples $(180 \mathrm{~Hz})$ emerge in the CA1 region, whereas the rhythm with a $110 \mathrm{~Hz}$ peak reflects a CA3 region-induced oscillation.

The hippocampal sharp wave reflects depolarization of CA1 pyramidal cells and interneurons by the CA3 afferents (Buzsaki et al., 1983). Large-amplitude sharp waves in the stratum radiatum are associated with a fast oscillatory field potential (ripple) at $140-200 \mathrm{~Hz}$ in stratum pyramidale (Buzsaki et al., 1992). The specific currents responsible for the ripple are believed to be synchronized somatic IPSPs interrupted by synchronous discharges of CA1 pyramidal neurons at every 5-6 msec ("mini population spikes"; Buzsaki, 1986; Ylinen et al., 1995; Draghun et al., 1998). Concurrent with the hippocampal sharp wave, ripples are present also in the subiculum, parasubiculum, and deep layers of the entorhinal cortex, but the ripple frequency is fastest in the CA1 region (Chrobak and Buzsaki, 1996). Fast ripple events in the CA1 region are preceded by increased neuronal discharges and field oscillation in the CA3 region (Buzsaki, 1986; Ylinen et al., 1995). However, our results revealed that the frequency of field oscillation in the CA3 region did not correlate with the CA1 ripple on a wave-by-wave basis. These findings support previous suggestions that fast ripples emerge locally in the CA1 region (Buzsaki et al., 1992; Draghun et al., 1998).

In contrast to the fast ripples, the slower oscillatory CA1 events (110 Hz peak) did correlate with the CA3 field and unit activity. Coordinated activity in the CA3 and CA1 regions is known to be present during both theta and gamma frequency activity (Bragin et al., 1995; Traub et al., 1996; Fisahn et al., 1998). In the slice preparation, bath application of the muscarinic agonist carbachol induces coherent network oscillation in the CA1 and CA3 regions. Depending on the concentration of the drug, the oscillation frequency can vary from the theta (Konopacki et al., 1997) to the gamma frequency band (Fisahn et al., 1998). These oscillations emerge in the CA3 network and are transferred to the CA1 region wave by wave. Similar transfer of the gamma activity has also been observed in vivo during theta-associated gamma activity (Bragin et al., 1995). We assume that field oscillations between large-amplitude CA1 ripples (i.e., $110 \mathrm{~Hz}$ peak events) are generated by a similar mechanism. In this framework, fast oscillatory events, emerging in different subregions of the CA3 field, rhythmically excite CA1 pyramidal neurons and interneurons, which faithfully follow the input. The observation that the oscillatory waves reversed either in the stratum oriens or radiatum support this contention, because subregions of the CA3 field differentially innervate the basal and apical dendrites of CA1 pyramidal cells 

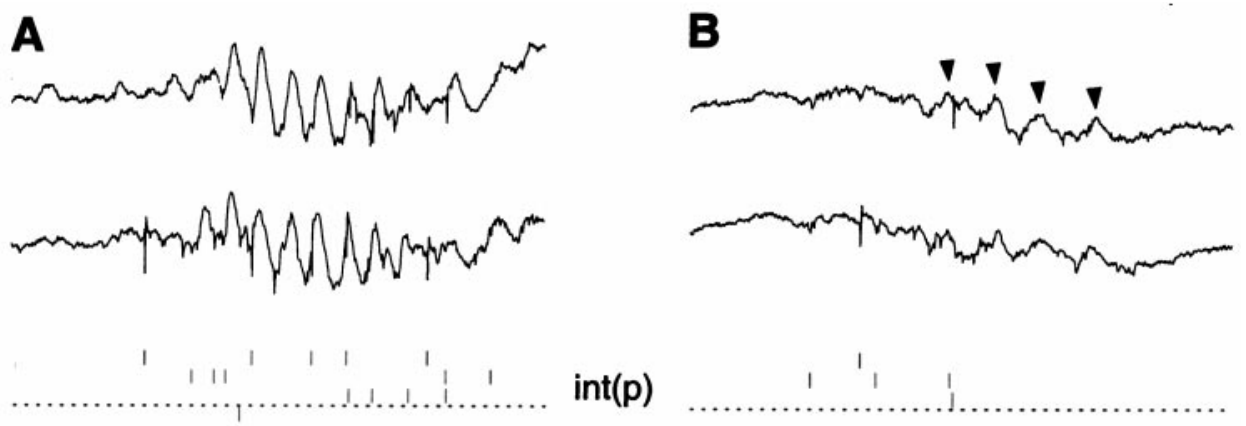

$\operatorname{int}(p)$

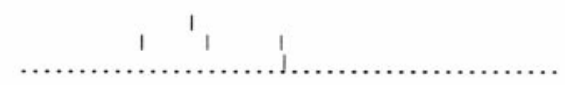

pyr
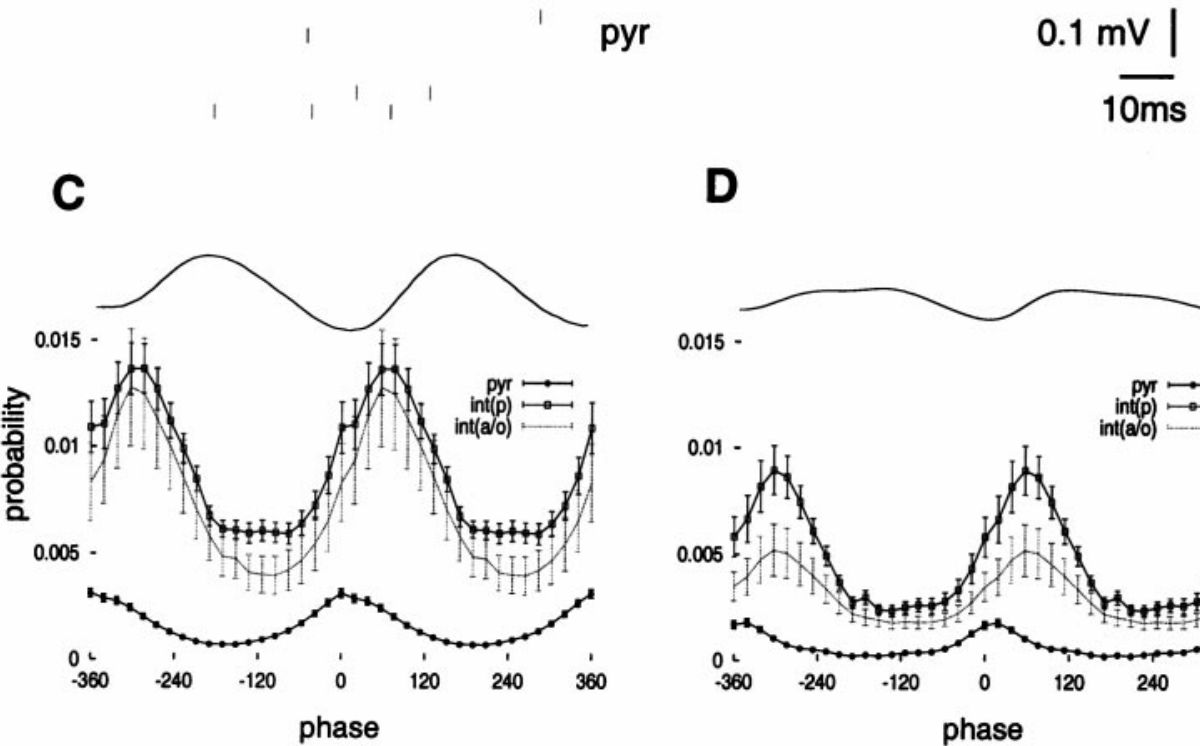

D

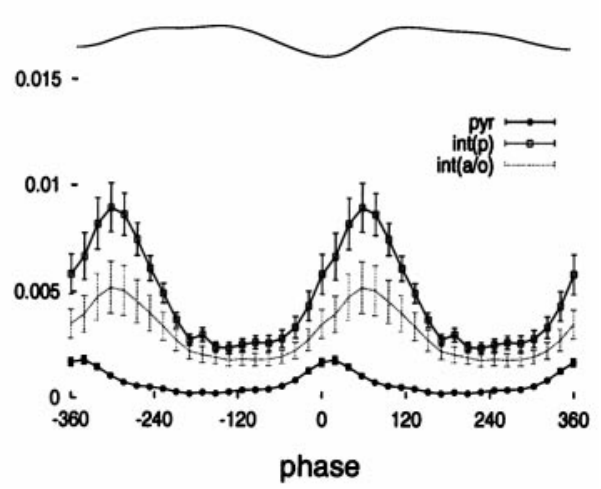

Figure 2. Unit and field activity in the CA1 region during fast oscillatory patterns. $A$, Sharp wave-associated fast ripple; $B$, lower-amplitude ( $<4$ SD) slow $(<130 \mathrm{~Hz})$ oscillatory event. Activity is displayed from two different tetrodes $(>300 \mu \mathrm{m}$ electrode distance, $1 \mathrm{~Hz}-5 \mathrm{kHz})$. Vertical ticks, Action potential times of isolated units. pyr, pyramidal cells; int $(p)$, pyramidal layer interneurons. $C$, $D$, Averaged phase histograms of unit firing during large-amplitude ( $>7 \mathrm{SD})$ ripple events $(C)$ and lower-amplitude $(<4 \mathrm{SD})$ oscillatory events $(D)$. Top traces, Averaged field oscillatory wave. Bottom histograms, Mean \pm SE phase histogram of pyramidal cells $(n=154)$, pyramidal layer interneurons $[$ int $(p) ; n=43]$, and neurons recorded in the alveus/stratum oriens $[\operatorname{int}(a / o) ; n=35]$. Note the lower discharge probability of neurons during the lower amplitude oscillations $(D)$. $C$ is replotted from Csicsvari et al. (1999).
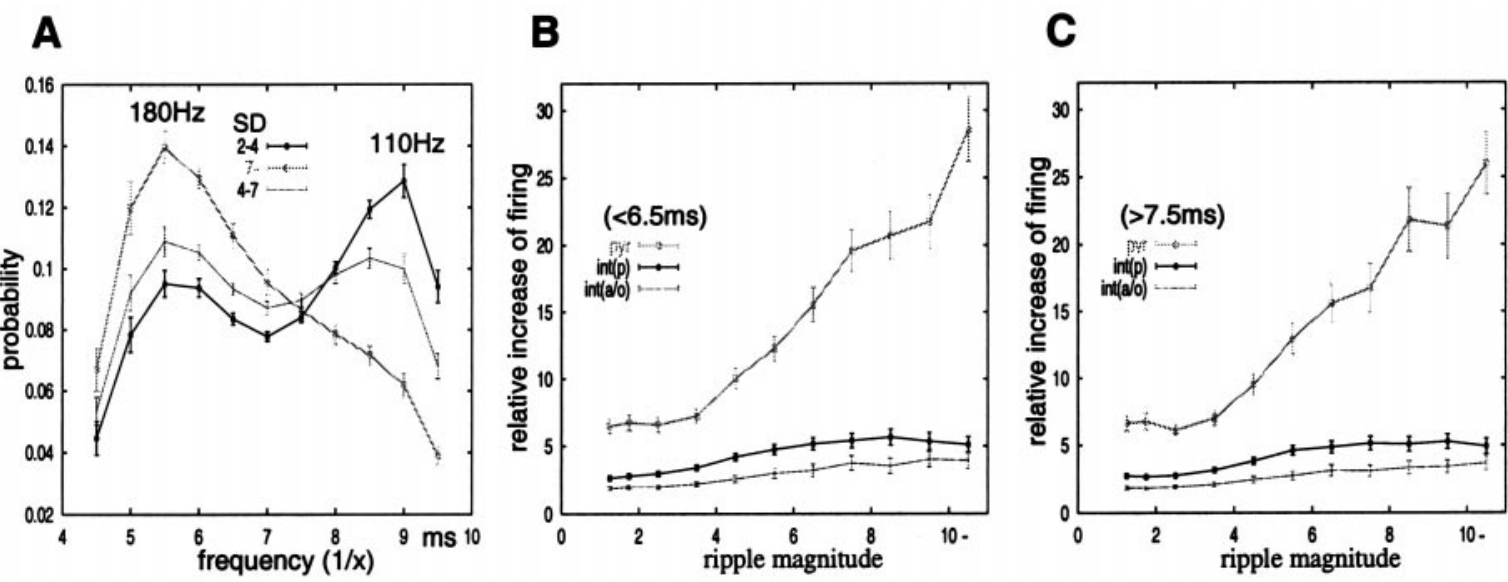

Figure 3. A, Frequency distribution (mean \pm SE) of the detected fast oscillatory patterns. Normalized histograms of small (2-4 SD)-, intermediate (4-7 $\mathrm{SD})$-, and large ( $>7 \mathrm{SD})$-amplitude patterns were averaged $(n=33)$. Note faster oscillation $(180 \mathrm{~Hz}$ peak) of the largest-amplitude events. $B$, $C$, Relative increase of unit firing during fast $(>140 \mathrm{~Hz} ; B)$ and slow $(<130 \mathrm{~Hz} ; C)$ oscillatory waves. For each recorded unit the increase of firing frequency was determined relative to the "flat" ( $<2 \mathrm{SD}$ ) baseline epochs ( $p y r, n=214$; $\operatorname{int}(p), n=43$; int $(a / o), n=214)$; ripple magnitude, peak amplitudes from 1 to $11 \mathrm{SD}$ from the baseline of the filtered $(80-250 \mathrm{~Hz})$ signal. 


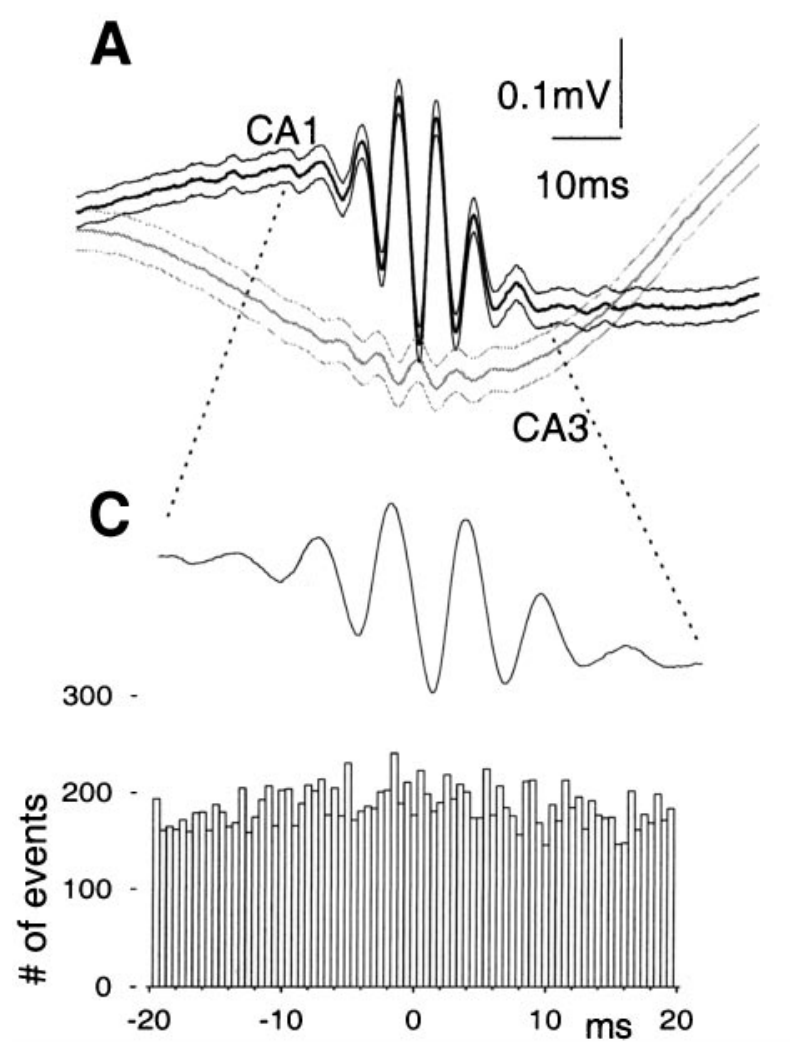

B


Figure 4. Relationship between CA1 and CA3 subfields. $A$, Fast, CA1 ripple-triggered field averages ( $>7 \mathrm{SD}) . B$, Slow CA1 wave triggered field averages $(<4 \mathrm{SD})$. Black thick line, Averaged field in CA1; gray thick line, averaged field in the CA3 region. Both CA1 and CA3 potentials were recorded in the pyramidal layer. Thin lines represent values 1 SEM. Note that the magnitude of the fast ripple-related (spurious) oscillation in the CA3 region is $<1$ SE. $C, D$, Cross-correlation between fast $(C)$ and slow $(D)$ CA1 oscillatory waves and spikes of a CA3 interneuron. Top trace, Averaged CA1 oscillatory wave (expanded parts of $A, B)$. Note the phase-locked discharge of the CA3 interneuron to the slow $(<130 \mathrm{~Hz})$ oscillatory field in CA1.

(Ishizuka et al., 1990; Li et al., 1994). However, in contrast to the theta-associated gamma, the frequency of the rhythm in the absence of theta is somewhat faster, and the oscillation is transient.

We hypothesize that locally emerging oscillations in the CA3 regions can be transferred to the CA1 pyramidal cells with high fidelity. On the other hand, when a large number of CA3 pyramidal cells discharge together, the strong depolarizing input to the CA1 neurons, as reflected by the field sharp wave, will induce a genuine new pattern, whose frequency is determined by the characteristics of the CA1 field rather than by its input. Thus, during sharp waves the fidelity of frequency transfer is low, and the CA1 region will forward a pattern to its targets, whose characteristics are determined by the CA1 network.

\section{REFERENCES}

Bland BH (1986) The physiology and pharmacology of hippocampal formation theta rhythms. Prog Neurobiol 26:1-54.

Bragin A, Jando G, Nadasdy Z, Hetke J, Wise K, and Buzsaki G (1995) Gamma $(40-100 \mathrm{~Hz})$ oscillation in the hippocampus of the behaving rat. J Neurosci 15:47-60.

Buzsaki G (1986) Hippocampal sharp waves: their origin and significance. Brain Res 398:242-252.

Buzsaki G, Leung LW, Vanderwolf CH (1983) Cellular bases of hippocampal EEG in the behaving rat. Brain Res 287:139-171.

Buzsaki G, Horvath Z, Urioste R, Hetke J, Wise K (1992) Highfrequency network oscillation in the hippocampus. Science 256:1025-1027.

Chrobak JJ, Buzsaki G (1996) High-frequency oscillations in the output networks of the hippocampal-entorhinal axis of the freely behaving rat. J Neurosci 16:3056-3066.
Csicsvari J, Hirase H, Czurko A, Mamiya A, Buzsaki G (1999) Oscillatory coupling of hippocampal pyramidal cells and interneurons in the behaving rat. J Neurosci 19:274-287.

Draguhn A, Traub RD, Schmitz D, Jefferys JG (1998) Electrical coupling underlies high-frequency oscillations in the hippocampus in vitro. Nature 394:189-192.

Fisahn A, Pike FG, Buhl EH, Paulsen O (1998) Cholinergic induction of network oscillations at $40 \mathrm{~Hz}$ in the hippocampus in vitro. Nature 394:186-189.

Ishizuka N, Weber J, Amaral DG (1990) Organization of intrahippocampal projections originating from CA3 pyramidal cells in the rat. J Comp Neurol 295:580-623.

Kandel A, Buzsaki G (1997) Cellular-synaptic generation of sleep spindles, spike-and-wave discharges, and evoked thalamocortical responses in the neocortex of the rat. J Neurosci 17:6783-6797.

Konopacki J, Golebiewski H, Eckersdorf B, Blaszczyk M, Grabowski R (1997) Theta-like activity in hippocampal formation slices: the effect of strong disinhibition of GABAA and GABAB receptors. Brain Res 775:91-98.

Li XG, Somogyi P, Ylinen A, Buzsaki G (1994) The hippocampal CA3 network: an in vivo intracellular labeling study. J Comp Neurol 339:181-208

Stewart M, Fox SE (1990) Do septal neurons pace the hippocampal theta rhythm? Trends Neurosci 13:163-168.

Traub RD, Whittington MA, Colling SB, Buzsaki G, Jefferys JG (1996) Analysis of gamma rhythms in the rat hippocampus in vitro and in vivo. J Physiol (Lond) 493:471-484.

Vanderwolf CH (1969) Hippocampal electrical activity and voluntary movement in the rat. Electroencephalogr Clin Neurophysiol 26:407-418.

Ylinen A, Bragin A, Nadasdy Z, Jando G, Szabo I, Sik A, Buzsaki G (1995) Sharp wave-associated high-frequency oscillation $(200 \mathrm{~Hz})$ in the intact hippocampus: network and intracellular mechanisms. J Neurosci 15:30-46. 